\title{
Use of a Bayesian model including QTL markers increases prediction reliability when test animals are distant from the reference population
}

\author{
Peipei Ma, ${ }^{1,2}$ Mogens S. Lund, ${ }^{2}$ Gert P. Aamand, ${ }^{3}$ and Guosheng Su ${ }^{2 *}$ \\ ${ }^{1}$ Department of Animal Science, School of Agriculture and Biology, Shanghai Jiao Tong University, Shanghai 200240, P.R. China \\ ${ }^{2}$ Center for Quantitative Genetics and Genomics, Department of Molecular Biology and Genetics, Aarhus University, DK-8830, Aarhus, Denmark \\ ${ }^{3}$ NAV Nordic Cattle Genetic Evaluation, DK-8200, Aarhus, Denmark
}

\section{ABSTRACT}

Relatedness between reference and test animals has an important effect on the reliability of genomic prediction for test animals. Because genomic prediction has been widely applied in practical cattle breeding and bulls have been selected according to genomic breeding value without progeny testing, the sires or grandsires of candidates might not have phenotypic information and might not be in the reference population when the candidates are selected. The objective of this study was to investigate the decreasing trend of the reliability of genomic prediction given distant reference populations, using genomic best linear unbiased prediction (GBLUP) and Bayesian variable selection models with or without including the quantitative trait locus (QTL) markers detected from sequencing data. The data used in this study consisted of 22,242 bulls genotyped using the $54 \mathrm{~K}$ SNP array from EuroGenomics. Among them, 1,444 Danish bulls born from 2006 to 2010 were selected as test animals. Different reference populations with varying relationships to test animals were created according to pedigree-based relationships. The reference individuals having a relationship with one or more test animals higher than 0.4 (scenario $\rho<0.4$ ), 0.2 ( $\rho$ $<0.2)$, or $0.1(\rho<0.1$, where $\rho=$ relationship coefficient) were removed from reference sets; these represented the distance between reference and test animals being 2 generations, 3 generations, and 4 generations, respectively. Imputed whole-genome sequencing data of bulls from Denmark were used to conduct a genomewide association study (GWAS). A small number of significant variants (QTL markers) from the GWAS were added to the array data. To compare the effects of different models, the basic GBLUP model, a Bayes-

Received October 9, 2018

Accepted March 31, 2019

*Corresponding author: guosheng.su@mbg.au.dk ian selection variable model, a GBLUP model with 2 components of genetic effects, and a Bayesian model with pooled array data and QTL markers were used for estimating genomic estimated breeding values (GEBV) of test animals. The reliability of genomic prediction decreased when the test animals were more generations away from the reference population. The reliability of genomic prediction was 0.461 for 1 generation away and 0.396 for 3 generations away, with the same number of individuals in the reference set, using a GBLUP model with chip markers only. The results showed that using the Bayesian method and QTL markers improved the reliability of genomic prediction in all scenarios of relationship between test and reference animals, in a range of $1.3 \%$ and $65.1 \%$ (4 generations away with only 841 individuals in the reference set). However, most gains were for predictions of milk yield and fat yield. There was little improvement for predictions of protein yield and mastitis, and no improvement for prediction of fertility, except for scenario $\rho<0.1$, in which there was a large improvement for predictions of all traits. On the other hand, models including more than $10 \%$ polygenic effect decreased prediction reliability when the relationship between test and reference animals was distant.

Key words: genomic prediction, distant relationship, Bayesian model, QTL markers

\section{INTRODUCTION}

Genomic prediction has been widely used in cattle breeding because it greatly decreases generation interval and attains high accuracy even for animals at early stages of life, compared with traditional pedigree-based BLUP (Schaeffer, 2006). Two hypotheses have been proposed for the success of genomic prediction. One is that the markers are in linkage disequilibrium (LD) with QTL, which affects specific traits (Goddard and Hayes, 2007). The other is that the markers could capture additive genetic relationships between individuals (Habier et al., 2007). Genomic best linear unbiased prediction 
(GBLUP), which is considered an efficient model, has been applied in most studies and in practical breeding (Erbe et al., 2012; Neves et al., 2014; Zhou et al., 2014). The model uses genomic relationships constructed with markers to replace the pedigree-based numerator relationships in the BLUP model (VanRaden, 2008; Yang et al., 2010). Many studies have reported that the relationship between reference population and test population is one of the key factors affecting prediction reliability (Habier et al., 2007, 2010; Gao et al., 2013).

The reliability of genomic prediction decreases when the relationship between the reference set and the test animals is weak (Gao et al., 2013; Wu et al., 2015b). Hence, it is necessary to include close relatives, especially the sire, in the reference population to ensure high accuracy of genomic prediction. However, the practical application of genomic prediction leads to situations in which test animals are 2 or more generations away from the reference population. For instance, if a bull is selected according to genomic prediction without progeny testing and subsequently is used as service sire as soon as it is sexually mature, this bull will not have its daughter's information available when its offspring are ready for selection (Habier et al., 2010). Therefore, it is necessary to investigate appropriate methods and strategies to slow the decrease in accuracy of genomic prediction in the situation of distant relationships between reference and test animals.

Previous studies have reported that the accuracy of genomic prediction using GBLUP was much lower than that of Bayesian methods in simulation data (Meuwissen et al., 2001), although both models were quite similar in real data ( $\mathrm{Su}$ et al., 2012). The reason could be that there are large numbers of QTL with small effects on particular traits of interest in real data but only a few QTL with large effects in simulation studies. However, the studies on model comparison in cattle populations are usually based on data in which reference and test animals have a relatively close relationship; typically sire and maternal grandsire are in the reference data. We expected that the accuracy of genomic prediction using Bayesian methods for scenarios of distant relationship between test and reference animals would perform better than GBLUP, because the genetic relationship decreased much faster than the decay of LD between QTL and markers (Habier et al., 2007).

Several studies have been conducted to improve the accuracy of genomic prediction by increasing the density of markers. Higher LD between markers and causative mutations in high-density chips is supposed to improve the reliability of genomic prediction. But only a slight increase in reliability was observed when using $777 \mathrm{~K}$ chip and sequence data, compared with using
54K chips (Su et al., 2012; van Binsbergen et al., 2015). However, the power for genome-wide association studies (GWAS) is improved using sequence data (Daetwyler et al., 2014; Wu et al., 2015a). Previous studies have showed that including QTL markers selected by GWAS using sequence data in the model could improve prediction reliability (Boichard et al., 2012; Brøndum et al., 2015; van den Berg et al., 2016; Ni et al., 2017). Assuming the detected QTL as causal variants, the effects of detected QTL will be consistent among animals across different generations; thus, the model including QTL should be more efficient for genomic prediction in the situation where test animals are less closely related to reference animals than in the situation where test animals are closer to reference animals.

Including the remaining polygenic effects other than SNP markers in the model could reduce the bias of genomic prediction without decreasing the prediction reliability in GBLUP (Hayes et al., 2009; Liu et al., 2011; Su et al., 2014a; Ma et al., 2015). However, the relevant studies in the literature have been based on data in which there was a strong relationship between test and reference animals (e.g., sire in reference population). So far there has been no report in the literature about the results of including the remaining polygenic effects in the model on prediction accuracy for distantly related livestock animals.

The objective of this study was to use EuroGenomics data to investigate genomic prediction for test animals that are 2 or more generations away from the reference animals, with focus on (1) inspecting the decay of reliability of genomic prediction with increasing distance between test animals and reference animals; (2) comparing GBLUP and Bayesian variable selection models for genomic prediction; (3) investigating the gain in prediction reliability by including QTL markers in the model; and (4) testing the results of including polygenic effects in the model on genomic prediction.

\section{MATERIALS AND METHODS}

\section{Data}

EuroGenomics Holstein data (Lund et al., 2011) from Germany, France, the Netherlands, and Nordic countries were used in this study. The data comprised 22,242 bulls. De-regressed proofs (DRP) in the Nordic scale for milk, fat, protein yield, fertility, and mastitis were used as phenotypes in this study. The animals were genotyped using Illumina BovineSNP50 BeadChip (54K; Illumina, San Diego, CA). After quality control for minor allele frequency (not less than 0.01), 44,397 autosome markers were used for genomic prediction. 


\section{Methods}

To validate the accuracy of genomic prediction, 1,444 Danish bulls born from 2006 to 2010 were used as the test set. Individuals born before 2006 were used as the reference set. The reference set included sires of 1,316 test individuals and grandsires of 1,434 test individuals. To mimic scenarios with different levels of relationship between test and reference animals, reference individuals having one or more test animals with a pedigreebased relationship higher than 0.4 (scenario $\rho<\mathbf{0 . 4}$ ), $0.2(\boldsymbol{\rho}<\mathbf{0 . 2})$, or $0.1(\boldsymbol{\rho}<\mathbf{0 . 1}$, where $\rho=$ relationship coefficient), respectively, were removed from the reference sets. Thus, scenarios with no restriction of $\rho, \rho$ $<0.4, \rho<0.2$, and $\rho<0.1$ represented the distance between reference and test animals being $1,2,3$, and 4 generations, respectively. This led to unequal numbers of bulls in different reference sets (Uneq_ $\mathbf{N}_{\text {ref }}$ ). To compare genomic prediction in different relationship scenarios with equal numbers of reference animals $\left(\mathbf{E q} \_\mathbf{N}_{\text {ref }}\right)$, the numbers of individuals in the reference set of $\rho<0.4$ and in the full reference set were reduced to equal the number in the reference set of $\rho<0.2$. This was done by randomly removing reference animals in the full reference set and the reference set of $\rho<0.2$. The procedure was repeated 5 times. Both $\mathrm{Eq} \_\mathrm{N}_{\text {ref }}$ and Uneq_ $\mathrm{N}_{\text {ref }}$ data sets were used to predict the breeding value $(\mathbf{B V})$ of test animals. The numbers of reference animals in each scenario are listed in Table 1.

\section{Methods for GWAS}

We performed GWAS using data from the Uneq_N $\mathrm{N}_{\text {ref }}$ reference without restriction of relationship. Because only Nordic data were allowed for GWAS by agreement, 3,114 Nordic bulls with imputed sequence data were available for GWAS in this study. The genotypes of these individuals were imputed from 50K to high density and then to whole-genome sequence level. The

Table 1. Numbers of reference individuals in each scenario ${ }^{1}$

\begin{tabular}{lcrcc}
\hline Data set & No & & & \\
& restriction & $\rho<0.4$ & $\rho<0.2$ & $\rho<0.1$ \\
\hline Uneq_N $_{\text {ref }}^{2}$ & 15,248 & 15,027 & 9,643 & 841 \\
Eq_N & 9,643 & 9,643 & 9,643 & $\mathrm{NA}^{4}$ \\
\hline
\end{tabular}

${ }^{1} \rho<0.4, \rho<0.2, \rho<0.1=$ relationships between all reference individuals and each test animal were restricted to be smaller than 0.4 , 0.2 , and 0.1 , respectively.

${ }^{2}$ Uneq_N $\mathrm{N}_{\text {ref }}=$ reference individuals born before 2006 .

${ }^{3} \mathrm{Eq} \_\mathrm{N}_{\text {ref }}=$ the size of reference set was reduced to 9,643 by randomly removing individuals from the reference sets.

${ }^{4}$ Not applicable. details of genotype imputation can be read in Höglund et al. (2014). The software EMMAX (Kang et al., 2010) was used for GWAS. The quality control criteria for markers used in GWAS were that the minor allele frequency should be larger than 0.005 , and the $P$-value of deviation from Hardy-Weinberg equilibrium should be larger than $10^{-7}$. In addition, SNP with effects of absolute value larger than twice the phenotype SD were removed. After quality control, 15,503,370 markers for milk yield, 15,457,837 markers for fat yield, 15,503,697 markers for protein yield, 15,374,515 markers for fertility, and 15,388,916 markers for mastitis were kept for GWAS analyses.

After GWAS analysis, several markers were selected as QTL markers to be used for genomic prediction according to the following criteria: (1) the $P$-values of these markers were smaller than 0.1 after Bonferroni correction; (2) at most the 10 most significant markers were selected within $1 \mathrm{Mb}$. In total, 956, 704, 802, 97, and 405 QTL markers were selected for the traits milk, fat, protein yields, fertility, and mastitis. The QTL markers were added into the $54 \mathrm{~K}$ marker sets, and all individuals were imputed to $54 \mathrm{~K}$ plus QTL markers with Beagle (Browning and Browning, 2009).

\section{Genomic Prediction Models}

A GBLUP model was implemented as the basic model. To investigate the effects of polygenic effects other than SNP markers, GBLUP models with polygenic effects accounting for 10,30 , and $50 \%$ of genetic variance were also used for genomic prediction. Alternative models are a Bayesian variable selection model and models including QTL markers.

\section{GBLUP Model}

The GBLUP model is as follows:

$$
\mathbf{y}=\mathbf{1} \mu+\mathbf{Z g}+\mathbf{e}
$$

where $\mathbf{y}$ is the vector of DRP in reference population, $\mu$ is the overall mean, $\mathbf{g}$ is the vector of $\mathbf{B V}, \mathbf{Z}$ is the design matrix for linking $\mathbf{g}$ to $\mathbf{y}$, and $\mathbf{e}$ is the vector of the random residuals. It is assumed that $\mathbf{g} \sim N\left(\mathbf{0}, \mathbf{G}_{\omega} \sigma_{g}^{2}\right)$, where $\sigma_{g}^{2}$ is the additive genetic variance; and $\mathbf{G}_{\omega}=\omega \mathbf{A}+(1-\omega) \mathbf{G}$, where $\mathbf{G}$ is the genomic relationship matrix using method 1 of VanRaden (2008), $\omega=0,0.1,0.3$, or 0.5 , to specify the polygenic effect accounting for genetic variance by $0,10,30$, or $50 \%$, respectively, and $\mathbf{A}$ is the numerator relationship coefficient matrix calculated using pedigree. It is also as- 
sumed that $\mathbf{e} \sim N\left(\mathbf{0}, \mathbf{D} \sigma_{e}^{2}\right)$, where $\sigma_{e}^{2}$ is the residual variance and $\mathbf{D}$ is a diagonal matrix with elements $d_{i i}=\left(1-r_{D R P}^{2}\right) / r_{D R P}^{2}$, for which $r_{D R P}^{2}$ is the reliability of individual DRP. The variance components were estimated using REML in DMU package (Madsen et al., 2010).

\section{Bayesian Mixture Model With or Without QTL Markers}

The Bayesian model is a mixture model with 4 normal distributions:

$$
\mathbf{y}=\mathbf{1} \mu+\mathbf{X q}+\mathbf{e}
$$

where $\mathbf{X}$ is the matrix of marker genotypes and $\mathbf{q}$ is the vector of SNP effects. The SNP in the Bayesian model without QTL markers included array data only, but the Bayesian model with QTL markers included both array data and QTL markers from GWAS. The model assumes that the distribution of a marker effect is a mixture of 4 normal distributions:

$$
q_{i} \sim \pi_{1} N\left(0, \sigma_{1}^{2}\right)+\pi_{2} N\left(0, \sigma_{2}^{2}\right)+\pi_{3} N\left(0, \sigma_{3}^{2}\right)+\pi_{4} N\left(0, \sigma_{4}^{2}\right),
$$

where $\pi_{k}(k=1,2,3$, or 4$)$ is the mixing proportion and $\sigma_{k}^{2}$ is the variance of distribution $k$. In this study, the mixing proportions were set to $\pi_{1}=0.889, \pi_{2}=0.1, \pi_{3}=0.01$, and $\pi_{4}=0.001$. The variances were estimated under the constraint $\sigma_{1}^{2}<\sigma_{2}^{2}<\sigma_{3}^{2}<\sigma_{4}^{2}$. The difference between this Bayesian model and BayesR (Erbe et al., 2012) is that $\sigma_{1}^{2}>0$ in this model, but $\sigma_{1}^{2}=0$ in BayesR. Gibbs sampling was implemented in the Bayesian model. The total samples were 50,000, and the first 10,000 were discarded as burn-in. Every 20th of the remaining 40,000 samples was used to calculate posterior statistics. Genomic estimated breeding values (GEBV) were calculated as the sum of all the marker effects.

\section{GBLUP Model with 2 Random Effects}

When QTL markers were included in the GBLUP model, the effects of the QTL markers and of the $54 \mathrm{~K}$ markers were taken as 2 separate components, resulting in the following GBLUP model:

$$
\mathbf{y}=\mathbf{1} \mu+\mathbf{Z}_{54 \mathrm{~K}} \mathbf{a}_{54 \mathrm{~K}}+\mathbf{Z}_{\mathrm{QTL}} \mathbf{a}_{\mathrm{QTL}}+\mathbf{e},
$$

where $\mathbf{a}_{54 \mathrm{~K}}$ and $\mathbf{a}_{\mathrm{QTL}}$ are the vectors of additive genetic values captured by the $54 \mathrm{~K}$ markers and QTL markers separately. It is assumed that $\mathbf{a}_{54 \mathrm{~K}} \sim N\left(\mathbf{0}, \mathbf{G}_{54 \mathrm{~K}} \sigma_{54 \mathrm{~K}}^{2}\right)$ and $\mathbf{a}_{\mathrm{QTL}} \sim N\left(\mathbf{0}, \mathbf{G}_{\mathrm{QTL}} \sigma_{Q T L}^{2}\right)$, where $\mathbf{G}_{54 \mathrm{~K}}$ and $\mathbf{G}_{\mathrm{QTL}}$ are the genomic relationship matrices constructed using $54 \mathrm{~K}$ markers and QTL markers, respectively, and $\sigma_{54 \mathrm{~K}}^{2}$ and $\sigma_{Q T L}^{2}$ are the genetic variances explained by the $54 \mathrm{~K}$ markers and QTL markers, respectively. Effects of all QTL markers were assumed to have the same variance in computing $\mathbf{G}_{\mathrm{QTL}} ; \mathbf{Z}_{54 \mathrm{~K}}$ and $\mathbf{Z}_{\mathrm{QTL}}$ were the design matrices for the 54K SNP and QTL marker effects. The GEBV were calculated as the sum of $\mathbf{a}_{54 \mathrm{~K}}$ and $\mathbf{a}_{\mathrm{QTL}}$. The variance components were estimated using REML in the DMU package (Madsen et al., 2010).

\section{Validation}

Prediction reliability was evaluated as the squared correlation of GEBV and DRP divided by the reliability of DRP (Su et al., 2014b) for test animals. The average reliabilities of DRP for test animals were 0.903 for yield traits, 0.701 for fertility, and 0.784 for mastitis. Bias was also investigated, using the regression coefficients of DRP on GEBV (Su et al., 2012); a prediction will be unbiased if the regression coefficient does not deviate significantly from 1 . Averages across 5 replicates were used for scenario Eq_N $\mathrm{N}_{\text {ref. }}$. Williams' test (Steiger, 1980) was used to check whether the reliabilities obtained from the other models were significantly different than the reliability obtained from the GBLUP model.

\section{RESULTS}

\section{Relationship Between Test Animals and Different Sets of Reference Animals}

The maximum relatedness (MAXR) of one reference individual to all the test individuals was chosen to measure the distance between reference and test sets. The average MAXR in different data sets is listed in Table 2. The average MAXR was slightly different between scenarios including or excluding the individuals with MAXR $>0.4$, but dropped considerably when restricting MAXR $<0.2$, and dropped greatly again with restricting MAXR $<0.1$.

\section{GWAS Results}

Manhattan plots for the GWAS are shown in Figure 1. The $-\log (P)$ value of the $D G A T$ gene was around 100 for milk yield and 150 for fat yield. To make the other 
Table 2. Average maximum relationships between reference data and test data in different scenarios ${ }^{1}$

\begin{tabular}{lcccc}
\hline Data set & $\begin{array}{c}\text { No } \\
\text { restriction }\end{array}$ & $\rho<0.4$ & $\rho<0.2$ & $\rho<0.1$ \\
\hline Uneq_N $_{\text {ref }}^{2}$ & 0.192 & 0.187 & 0.147 & 0.077 \\
Eq_N & 0.192 & 0.187 & 0.147 & $\mathrm{NA}^{4}$ \\
\hline
\end{tabular}

${ }^{1} \rho<0.4, \rho<0.2, \rho<0.1=$ relationships between all reference individuals and each test animal were restricted to be smaller than 0.4, 0.2 , and 0.1 , respectively.

${ }^{2}$ Uneq_ $\mathrm{N}_{\text {ref }}=$ reference individuals born before 2006 .

${ }^{3} \mathrm{Eq} \_\mathrm{N}_{\text {ref }}=$ size of reference set was reduced to 9,643 by randomly removing individuals from the reference sets. Results were averaged across 5 replicates.

${ }^{4}$ Not applicable.

QTL regions visible, we set the ranges of the $y$-axes as 0 to 20 for milk yield and 0 to 30 for fat and protein yield. More than 700 markers were selected as QTL markers for most production traits, although only 405 markers were selected for mastitis and 97 for fertility.

\section{Genomic Prediction Using GBLUP}

The reliabilities of genomic prediction for different scenarios are shown in Tables 3 and 4 . For a distance of one generation, the prediction reliabilities using the Uneq_ $\mathrm{N}_{\text {ref }}$ and Eq_N $\mathrm{N}_{\text {ref }}$ data sets is 0.51 and 0.46 , on average of the 5 traits, respectively. The prediction reliability was improved by approximately $5 \%$ using more reference individuals (data set Uneq_N $\mathrm{N}_{\text {ref }}$ ), which indicated that the size of reference population was quite important. The regression coefficients of DRP on GEBV in all scenarios using Uneq_N $\mathrm{N}_{\text {ref }}$ and Eq_N $\mathrm{N}_{\text {ref }}$ were similar (Tables 5 and 6 ).

In both scenarios Uneq_ $\mathrm{N}_{\text {ref }}$ and Eq_N $\mathrm{N}_{\text {ref }}$, the reliabilities decreased when the relationships between reference individuals and test individuals were weak. Very low reliability (0.093 averaged over 5 traits) was observed in relationship scenarios of $\rho<0.1$, which could be due not only to a weak genetic relationship but also to a very small reference population size (841 animals). A large decrease of regression coefficients was observed for relationship scenarios of $\rho<0.1$, although the decrease was relatively small for other relationship scenarios.

\section{Genomic Prediction Using Other Models}

Compared with GBLUP, the proportions of reliability changed using the other methods listed in the last
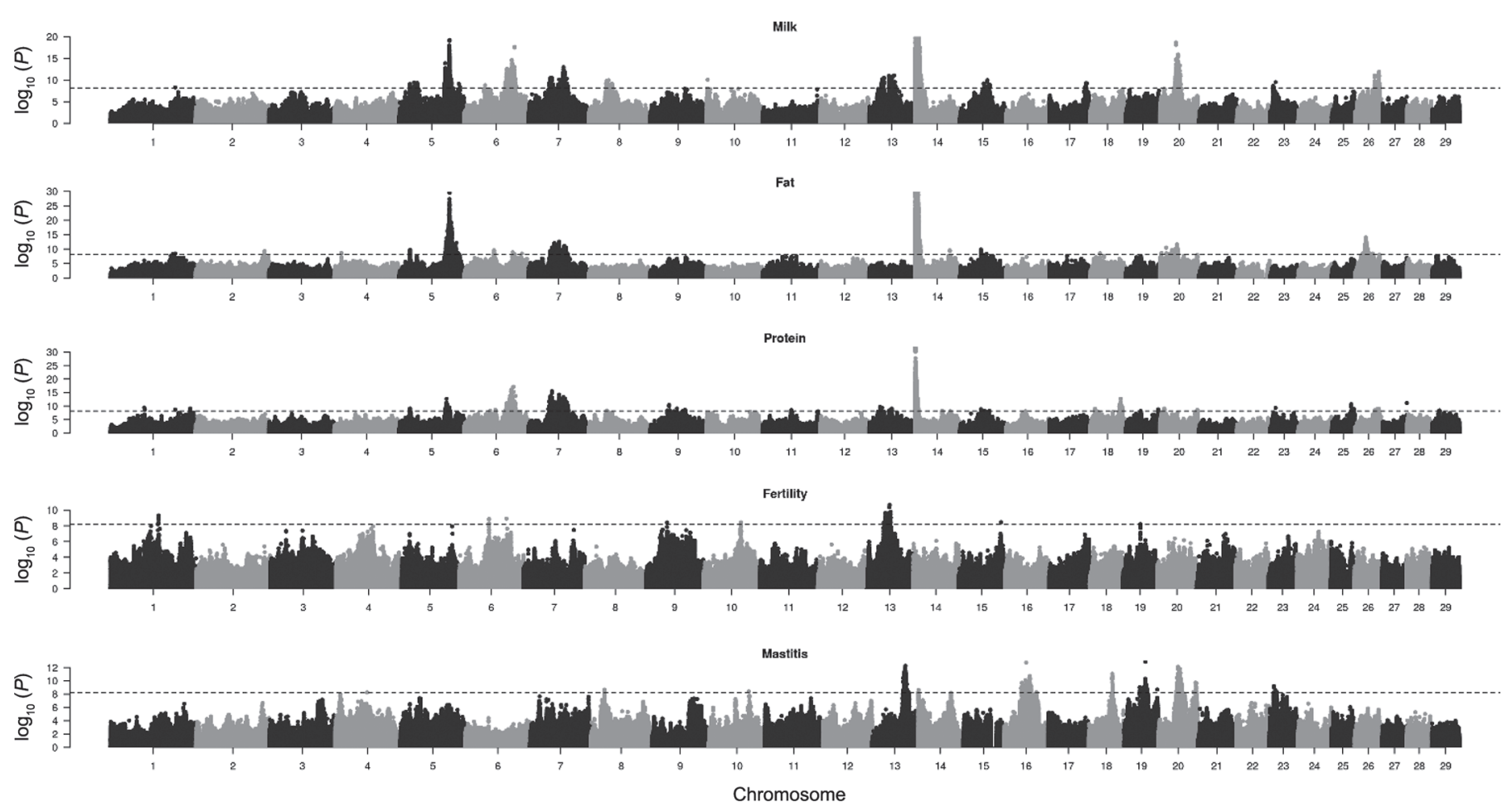

Figure 1. Manhattan plots for association of SNP for all 5 traits. Threshold line indicates genome-wide significance level $(P$-values set to 0.01 after Bonferroni correction). Ranges of $y$-axes have been set to 0 to 20 for milk yield and 0 to 30 for fat and protein yield, to make most QTL regions visible. However, the peak of the DGAT gene, which is on chromosome 14, was truncated. 
column in Tables 3 and 4 . When only $10 \%$ polygenic effects were included in the GBLUP model, the reliability was similar to those from GBLUP without polygenic effects, but the regression coefficient was closer to 1 regardless of the relationship and size of reference population. The GBLUP models with higher proportions of polygenic effects showed lower reliabilities and less bias (deviation of regression coefficient from 1). The GBLUP with $30 \%$ polygenic effects obtained slightly lower reliability of genomic prediction, and the GBLUP with $50 \%$ polygenic effects obtained much lower reliability of genomic prediction, compared with GBLUP. In addition, reducing the size of the reference population led to a greater decrease of reliability using GBLUP with large proportions of polygenic effects. For example, the changes in reliability using data set Uneq_ $\mathrm{N}_{\text {ref }}$ for the scenario of one generation away were $0.5,0$, and $-1.9 \%$ for GBLUP with 10, 30, and $50 \%$ polygenic effect, respectively, and the changes in reliability using Eq_ $\mathrm{N}_{\text {ref }}$ were $0.2,-0.9$, and $-3.8 \%$, respectively.

Using the Bayesian method improved prediction reliability no matter the relationship between reference and test animals. Bayesian methods performed better than GBLUP for almost all traits, especially for fat yield. The regression coefficients for the Bayesian method were similar to those of GBLUP.

The GBLUP including QTL markers performed better than GBLUP using only chip SNP for milk yield, fat yield, and mastitis in most cases, although the gains from QTL markers in prediction reliabilities for protein yield and fertility were small. The regression coefficients were a little closer to 1 for GBLUP including QTL markers, compared with GBLUP using only chip $\mathrm{SNP}$, in most scenarios.

Table 3. Reliabilities of genomic prediction for scenario Uneq_ $\mathrm{N}_{\text {ref }}{ }^{1}$

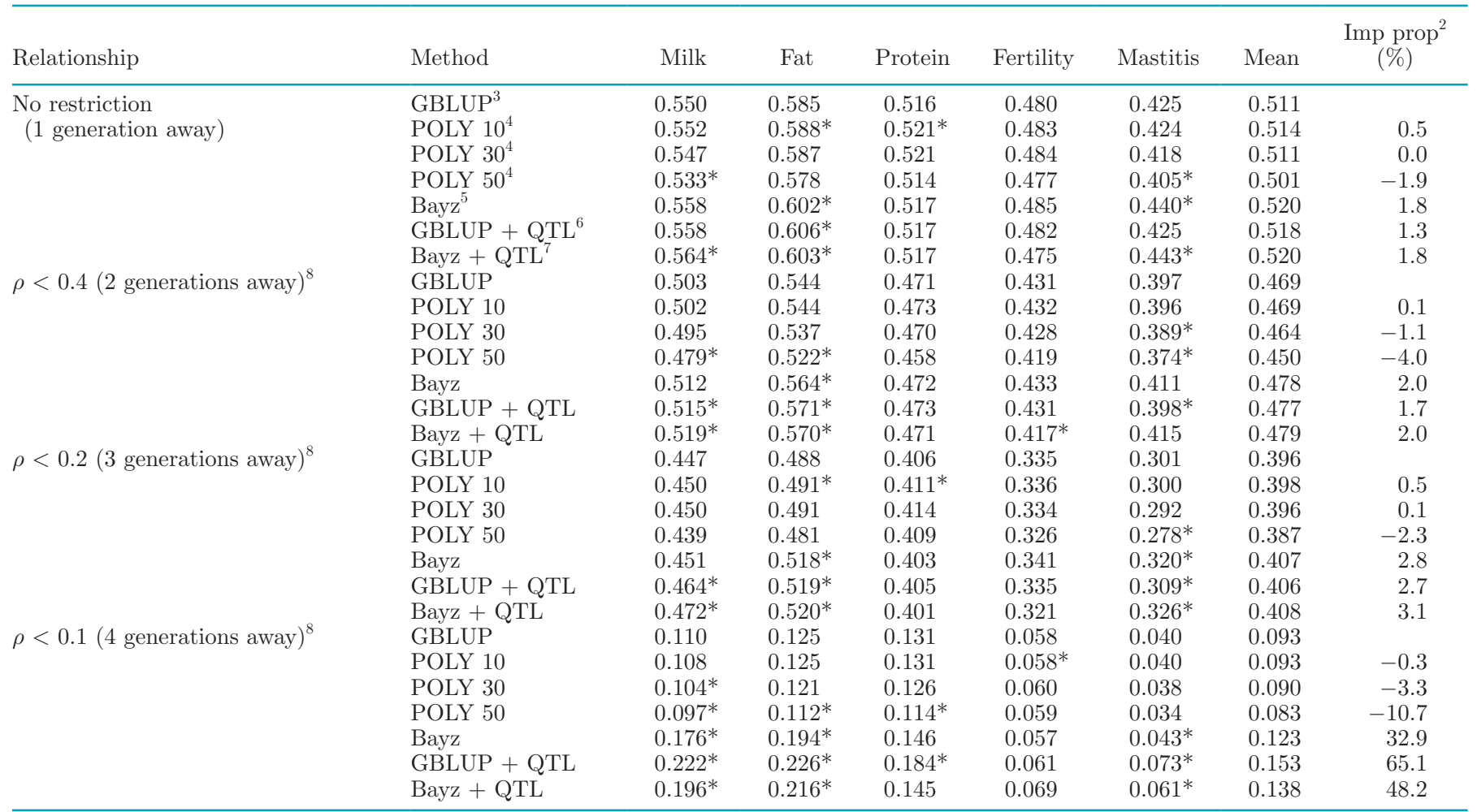

\footnotetext{
${ }^{1}$ Uneq_ $\mathrm{N}_{\text {ref }}=$ reference data sets included all reference individuals available.

${ }^{2} \mathrm{Imp}$ prop $=$ improved proportion compared with basic GBLUP method.

${ }^{3}$ GBLUP $=$ genomic best linear unbiased prediction.

${ }^{4}$ POLY 10, POLY 30, POLY 50 = GBLUP with 10, 30, and 50\% polygenic effects, respectively.

${ }^{5}$ Bayz $=$ Bayesian mixture model with 4 normal distributions with array data.

${ }^{6}$ GBLUP + QTL $=$ the effects of QTL markers and 54K markers were taken as 2 separate components in GBLUP.

${ }^{7}$ Bayz + QTL $=$ Bayesian mixture model with 4 normal distributions, with both array data and QTL markers.

${ }^{8} \rho<0.4, \rho<0.2, \rho<0.1=$ relationships between reference individuals and test animals were restricted to be smaller than $0.4,0.2$, and 0.1 , respectively.

* Reliability of genomic prediction differs significantly from that derived from GBLUP model.
} 
When QTL markers were included, the Bayesian method and GBLUP obtained similar reliability of genomic prediction. However, the regression coefficients using Bayesian methods deviated more from 1 than did GBLUP including QTL markers.

\section{DISCUSSION}

This study investigated the decay trend in prediction reliability when the relationship between reference and test set was distant. Strategies including using a Bayesian method and adding QTL markers to the chip data were tested. The results showed that these strategies could be used to improve reliabilities of genomic prediction, regardless of the degree of relationship between test and reference animals. On the other hand, models with polygenic effects could reduce bias of genomic prediction regardless of the relationship between reference and test populations or the size of the reference population. However, the reliability of genomic prediction using models with polygenic effects decreased compared with GBLUP without polygenic effects when the distance between reference and test animals increased.

In this study, we used maximum relationships to measure the distance between reference set and test animals. The maximum relatedness has been proved to be a reasonable criterion for measuring the relationship in genomic prediction studies (Clark et al., 2012) and fit the aim of this study, which is to investigate the change of genomic predictive ability when the sires, grandsires, or great-grandsires of test animals were not in the reference population. We did not simply remove sires or grandsires because high connectedness between farms from the sampled countries and overlapping generations of cattle may generate close relationships similar to those of sires or grandsires with test animals, which would be different from situations in which test animals were 2 or more generations away from reference animals.

The relationship between reference and test population has an effect on prediction reliability. This could be explained by the fact that more strongly related individuals provided more information for the prediction of GEBV (Habier et al., 2010). Decreasing reliability of genomic prediction because of distant relationships between test and reference sets could be compensated for by increasing the size of the reference population. This could be explained by index theory. To some extent, more data can balance a lower relationship coefficient. An increase of 0.039 in reliability was observed when the relationship was increased from $\rho<0.4$ to no restriction, with the same number of individuals $(9,643)$ in

Table 4. Reliabilities of genomic prediction for scenario Eq__ $\mathrm{N}_{\text {ref }}{ }^{1}$

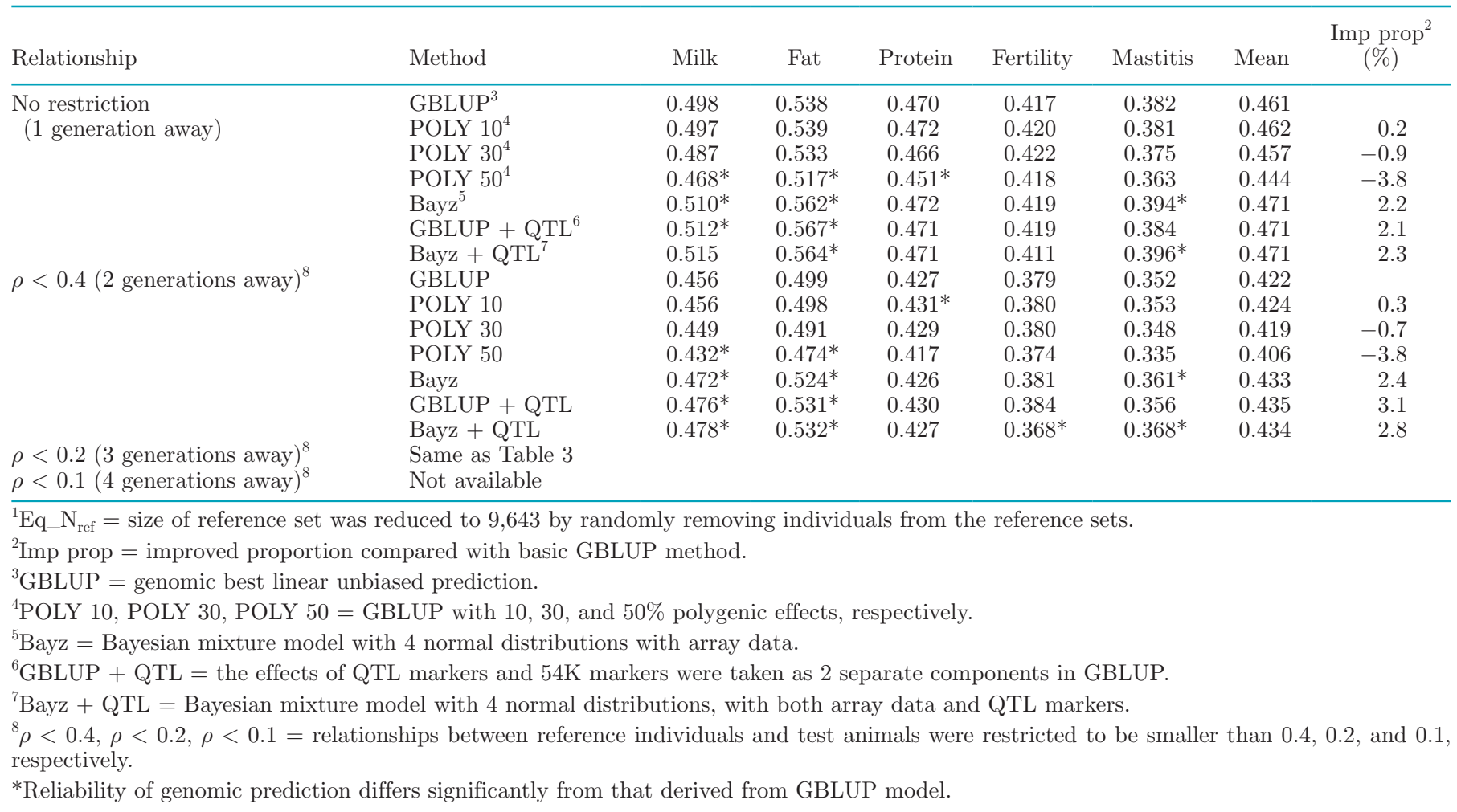


the reference population, using GBLUP. When the size of the reference population was increased from 9,643 (Eq_N $\left.\mathrm{N}_{\text {ref }}\right)$ for scenario $\rho<0.4$ to 15,027 (Uneq_N $\left.\mathrm{N}_{\text {ref }}\right)$ for scenario $\rho<0.4$, the reliability of genomic prediction using GBLUP increased by 0.047 . The results indicated that both the size of the reference population and the relationship between reference and test populations were important.

When QTL markers were not included, Bayesian methods outperformed GBLUP for predictions of fat yield and milk yield more than for predictions of protein yield, mastitis, or fertility. The reason could be that fat yield and milk yield had more highly associated QTL markers across the genome (Figure 1), which is consistent with the finding that variable selection methods performed better than GBLUP for traits affected by genes of moderate to large effects (VanRaden et al., 2009; Legarra et al., 2011). The GBLUP model treated all markers with the same variance, which relies more upon the genomic relationship constructed with markers than upon the LD between QTL and markers.
The Bayesian methods could capture the LD between QTL and markers. Because the LD between QTL and markers decayed slowly compared with the decrease of relationship between relatives (Habier et al., 2007), Bayesian methods performed better than GBLUP for most scenarios. There was a slight increase for scenarios with distant relationships between reference and candidate animals. Similar results were obtained from a previous study (Habier et al., 2010); however, the earlier results indicated that BayesB outperformed GBLUP with increasing training size. We observed discrepant results in our study: when the training size was larger, the superiority of the Bayesian method over GBLUP was smaller, regardless of absolute value or relative improvement. One possible reason for this discrepancy might be that the size of the reference population in the earlier study was small, with only 2,096 individuals included.

When QTL markers detected using GWAS based on sequence data of the reference population were included in the model, the GBLUP model with 2 components of

Table 5. Regression coefficient of genomic prediction for scenario Uneq_ $\mathrm{N}_{\text {ref }}{ }^{1}$

\begin{tabular}{|c|c|c|c|c|c|c|c|}
\hline Relationship & Method & Milk & Fat & Protein & Fertility & Mastitis & Mean \\
\hline \multirow{7}{*}{$\begin{array}{l}\text { No restriction } \\
\text { (1 generation away) }\end{array}$} & GBLUP $^{2}$ & 0.849 & 0.860 & 0.788 & 0.950 & 0.879 & 0.865 \\
\hline & POLY $10^{3}$ & 0.867 & 0.873 & 0.807 & 0.979 & 0.898 & 0.885 \\
\hline & POLY $30^{3}$ & 0.902 & 0.907 & 0.846 & 1.035 & 0.950 & 0.928 \\
\hline & POLY $50^{3}$ & 0.943 & 0.954 & 0.891 & 1.101 & 1.017 & 0.981 \\
\hline & Bayz ${ }^{4}$ & 0.843 & 0.865 & 0.787 & 0.944 & 0.887 & 0.865 \\
\hline & GBLUP $+\mathrm{QTL}^{5}$ & 0.854 & 0.868 & 0.785 & 0.949 & 0.877 & 0.867 \\
\hline & Bayz + QTL ${ }^{6}$ & 0.851 & 0.859 & 0.787 & 0.932 & 0.893 & 0.865 \\
\hline \multirow[t]{7}{*}{$\rho<0.4$ (2 generations away $)^{7}$} & GBLUP & 0.819 & 0.823 & 0.753 & 0.955 & 0.828 & 0.836 \\
\hline & POLY 10 & 0.836 & 0.833 & 0.772 & 0.988 & 0.846 & 0.855 \\
\hline & POLY 30 & 0.877 & 0.865 & 0.814 & 1.058 & 0.898 & 0.902 \\
\hline & POLY 50 & 0.925 & 0.909 & 0.862 & 1.149 & 0.965 & 0.962 \\
\hline & Bayz & 0.815 & 0.833 & 0.756 & 0.948 & 0.834 & 0.837 \\
\hline & GBLUP + QTL & 0.829 & 0.838 & 0.750 & 0.950 & 0.828 & 0.839 \\
\hline & Bayz + QTL & 0.824 & 0.833 & 0.757 & 0.925 & 0.841 & 0.836 \\
\hline \multirow[t]{7}{*}{$\rho<0.2(3 \text { generations away })^{7}$} & GBLUP & 0.826 & 0.857 & 0.742 & 0.912 & 0.817 & 0.831 \\
\hline & POLY 10 & 0.848 & 0.876 & 0.766 & 0.940 & 0.835 & 0.853 \\
\hline & POLY 30 & 0.900 & 0.923 & 0.820 & 1.006 & 0.888 & 0.908 \\
\hline & POLY 50 & 0.969 & 0.995 & 0.891 & 1.104 & 0.969 & 0.986 \\
\hline & Bayz & 0.814 & 0.869 & 0.734 & 0.902 & 0.838 & 0.831 \\
\hline & GBLUP + QTL & 0.830 & 0.870 & 0.725 & 0.911 & 0.827 & 0.833 \\
\hline & Bayz + QTL & 0.838 & 0.866 & 0.736 & 0.863 & 0.846 & 0.830 \\
\hline \multirow[t]{7}{*}{$\rho<0.1$ (4 generations away $)^{7}$} & GBLUP & 0.642 & 0.779 & 0.628 & 0.598 & 0.588 & 0.647 \\
\hline & POLY 10 & 0.666 & 0.824 & 0.655 & 0.633 & 0.614 & 0.678 \\
\hline & POLY 30 & 0.736 & 0.935 & 0.732 & 0.724 & 0.688 & 0.763 \\
\hline & POLY 50 & 0.848 & 1.094 & 0.849 & 0.867 & 0.792 & 0.890 \\
\hline & Bayz & 0.689 & 0.800 & 0.616 & 0.570 & 0.588 & 0.653 \\
\hline & GBLUP + QTL & 0.727 & 0.837 & 0.650 & 0.594 & 0.734 & 0.708 \\
\hline & Bayz + QTL & 0.694 & 0.827 & 0.595 & 0.573 & 0.728 & 0.683 \\
\hline
\end{tabular}

\footnotetext{
${ }^{1}$ Uneq_N $\mathrm{N}_{\text {ref }}=$ reference data sets included all reference individuals available.

${ }^{2}$ GBLUP $=$ Genomic best linear unbiased prediction.

${ }^{3}$ POLY 10, POLY 30, POLY 50 = GBLUP with 10, 30, and 50\% polygenic effects, respectively.

${ }^{4}$ Bayz $=$ Bayesian mixture model with 4 normal distributions with array data.

${ }^{5} \mathrm{GBLUP}+\mathrm{QTL}=$ the effects of QTL markers and $54 \mathrm{~K}$ markers were taken as 2 separate components in GBLUP.

${ }^{6}$ Bayz + QTL $=$ Bayesian mixture model with 4 normal distributions, with both array data and QTL markers.

${ }^{7} \rho<0.4, \rho<0.2, \rho<0.1=$ relationships between reference individuals and test animals were restricted to be smaller than $0.4,0.2$, and 0.1 , respectively.
} 
Table 6. Regression coefficients of genomic prediction for scenario Eq_ $\mathrm{N}_{\text {ref }}{ }^{1}$

\begin{tabular}{|c|c|c|c|c|c|c|c|}
\hline Relationship & Method & Milk & Fat & Protein & Fertility & Mastitis & Mean \\
\hline \multirow{5}{*}{$\begin{array}{l}\text { No restriction } \\
(1 \text { generation away })\end{array}$} & POLY $10^{3}$ & 0.853 & 0.870 & 0.795 & 0.954 & 0.875 & 0.869 \\
\hline & POLY $30^{3}$ & 0.887 & 0.904 & 0.829 & 1.010 & 0.927 & 0.911 \\
\hline & Bayz $^{4}$ & 0.829 & 0.861 & 0.773 & 0.916 & 0.859 & 0.847 \\
\hline & GBLUP + QTL $^{5}$ & 0.841 & 0.863 & 0.778 & 0.967 & 0.858 & 0.862 \\
\hline & Bayz + QTL ${ }^{6}$ & 0.835 & 0.853 & 0.772 & 0.906 & 0.865 & 0.846 \\
\hline \multirow{5}{*}{$\rho<0.4(2 \text { generations away })^{7}$} & POLY 30 & 0.868 & 0.866 & 0.801 & 1.044 & 0.900 & 0.896 \\
\hline & POLY 50 & 0.922 & 0.916 & 0.853 & 1.142 & 0.970 & 0.961 \\
\hline & Bayz & 0.806 & 0.820 & 0.731 & 0.928 & 0.825 & 0.822 \\
\hline & GBLUP + QTL & 0.812 & 0.821 & 0.733 & 0.931 & 0.827 & 0.825 \\
\hline & Bayz + QTL & 0.812 & 0.823 & 0.736 & 0.903 & 0.834 & 0.822 \\
\hline$\rho<0.2(3 \text { generations away })^{7}$ & Same as Table 5 & & & & & & \\
\hline$\rho<0.1$ (4 generations away) ${ }^{7}$ & Not available & & & & & & \\
\hline
\end{tabular}

${ }^{1} \mathrm{Eq} \_\mathrm{N}_{\text {ref }}=$ size of reference set was reduced to 9,643 by randomly removing individuals from the reference sets.

${ }^{2}$ GBLUP $=$ genomic best linear unbiased prediction.

${ }^{3}$ POLY 10, POLY 30, POLY $50=$ GBLUP with 10,30 , and $50 \%$ polygenic effects, respectively.

${ }^{4}$ Bayz $=$ Bayesian mixture model with 4 normal distributions with array data.

${ }^{5}$ GBLUP + QTL $=$ the effects of QTL markers and 54K markers were taken as 2 separate components in GBLUP.

${ }^{6}$ Bayz + QTL $=$ Bayesian mixture model with 4 normal distributions, with both array data and QTL markers.

${ }^{7} \rho<0.4, \rho<0.2, \rho<0.1=$ relationships between reference individuals and test animals were restricted to be smaller than $0.4,0.2$, and 0.1 , respectively.

genetic effects improved the accuracy of genomic prediction. Similar to Bayesian methods, the proportion of improvement for 5 traits occurred in the order fat yield $>$ milk yield $>$ mastitis $>$ protein yield $>$ fertility. The reason was that the detected markers were likely close to the causal mutations, and the 2-component model allowed larger weight on the QTL component (Brøndum et al., 2015). The disadvantage of this method was that a GWAS with high power needed to be conducted before implementation of genomic prediction. If the QTL markers have been found in the target population once, the computation load will not be an issue. However, it would be a good strategy to update the QTL markers when the size of the reference set has a large increase. In the scenario of $\rho<0.1$, the reliability of genomic prediction was improved with a high proportion for all traits when QTL markers were included in the model. A realistic situation corresponding to this scenario might be conducting genomic selection for other populations of the same breed - for instance, if a commercial company provides GEBV for customers all over the world. Results from this study suggest that the reliability of genomic prediction might be improved using QTL markers from the same breed for populations with small numbers of reference individuals. In reality, the data set used for GWAS should be the same as the reference set used for genomic prediction. However, we used the largest data set possible to conduct GWAS, because quite a small number of animals were chosen in most scenarios. Therefore, the improvement could be weakened in reality if QTL markers were obtained according to GWAS in the involved population. Alternatively, we could use QTL markers from public databases to assist in getting precise causal information.

Polygenic effects were included in the GBLUP model to account for genetic variation that cannot be explained by markers and to reduce the bias of genomic prediction (Liu et al., 2011; Ma et al., 2015). Applying this model to predict $\mathrm{BV}$ for test animals led to a decrease in the reliability of genomic prediction when the relationship between test and reference animals was distant. The reason could be that, through pedigree relationship, only family information can contribute to EBV of test animals, and family information decays at a rate of $50 \%$ per generation. The regression coefficients were improved with polygenic effects for all scenarios in this study. The results of our study illustrate that a small proportion $(10 \%)$ of polygenic effects can be included in the model to decrease bias without a decrease in the accuracy of genomic prediction when test animals are distant from reference populations.

\section{CONCLUSIONS}

Because of the wide use of genomic selection in dairy cattle, the sires and even the grandsires of test animals may not have phenotype information when the test animals are ready for selection using genotype informa- 
tion. In our study, we found that the Bayesian method and QTL markers increased the reliability of genomic prediction averaged across 5 traits, regardless of the relationship between reference and test animals. The main improvement was found for milk yield and fat yield, little for mastitis and protein yield, and none for fertility. Large proportions of polygenic effects in the model would decrease the reliability of genomic prediction when the reference and test animals are distantly related. However, a small proportion (10\%) of polygenic effects could be used to decrease bias of genomic prediction.

\section{ACKNOWLEDGMENTS}

This work was supported by the Genomics in Herds project, financed by VikingGenetics and Nordic Cattle Genetic Evaluation. We thank the editor and two reviewers whose comments and suggestions have greatly improved this manuscript.

\section{REFERENCES}

Boichard, D., F. Guillaume, A. Baur, P. Croiseau, M. N. Rossignol, M. Y. Boscher, T. Druet, L. Genestout, J. J. Colleau, L. Journaux, V. Ducrocq, and S. Fritz. 2012. Genomic selection in French dairy cattle. Anim. Prod. Sci. 52:115-120. https://doi.org/10.1071/ AN11119.

Brøndum, R. F., G. Su, L. Janss, G. Sahana, B. Guldbrandtsen, D. Boichard, and M. S. Lund. 2015. Quantitative trait loci markers derived from whole genome sequence data increases the reliability of genomic prediction. J. Dairy Sci. 98:4107-4116. https://doi.org/ 10.3168/jds.2014-9005.

Browning, B. L., and S. R. Browning. 2009. A unified approach to genotype imputation and haplotype-phase inference for large data sets of trios and unrelated individuals. Am. J. Hum. Genet. 84:210-223. https://doi.org/10.1016/j.ajhg.2009.01.005.

Clark, S.A., J.M. Hickey, H.D. Daetwyler, and J.H. van der Werf 2012. The importance of information on relatives for the prediction of genomic breeding values and the implications for the makeup of reference data sets in livestock breeding schemes. Genet. Sel. Evol. 44:4. https://doi.org/10.1186/1297-9686-44-4.

Daetwyler, H. D., A. Capitan, H. Pausch, P. Stothard, R. van Binsbergen, R. F. Brøndum, X. Liao, A. Djari, S. C. Rodriguez, C. Grohs, D. Esquerré, O. Bouchez, M.-N. Rossignol, C. Klopp, D. Rocha, S. Fritz, A. Eggen, P. J. Bowman, D. Coote, A. J. Chamberlain, C. Anderson, C. P. VanTassell, I. Hulsegge, M. E. Goddard, B. Guldbrandtsen, M. S. Lund, R. F. Veerkamp, D. A. Boichard, R. Fries, and B. J. Hayes. 2014. Whole-genome sequencing of 234 bulls facilitates mapping of monogenic and complex traits in cattle. Nat. Genet. 46:858-865. https://doi.org/10.1038/ng.3034.

Erbe, M., B.J. Hayes, L.K. Matukumalli, S. Goswami, P.J. Bowman, C.M. Reich, B.A. Mason, and M.E. Goddard. 2012. Improving accuracy of genomic predictions within and between dairy cattle breeds with imputed high-density single nucleotide polymorphism panels. J. Dairy Sci. 95:4114-4129. https://doi.org/10.3168/jds 2011-5019.

Gao, H., G. Su, L. Janss, Y. Zhang, and M. S. Lund. 2013. Model comparison on genomic predictions using high-density markers for different groups of bulls in the Nordic Holstein population. J. Dairy Sci. 96:4678-4687. https://doi.org/10.3168/jds.2012-6406.

Goddard, M. E., and B. J. Hayes. 2007. Genomic selection. J. Anim. Breed. Genet. 124:323-330. https://doi.org/10.1111/j.1439-0388 .2007.00702.x.
Habier, D., R. L. Fernando, and J. C. M. Dekkers. 2007. The impact of genetic relationship information on genome-assisted breeding values. Genetics 177:2389-2397. https://doi.org/10.1534/genetics .107.081190.

Habier, D., J. Tetens, F.-R. Seefried, P. Lichtner, and G. Thaller. 2010. The impact of genetic relationship information on genomic breeding values in German Holstein cattle. Genet. Sel. Evol. 42:5. https: //doi.org/10.1186/1297-9686-42-5.

Hayes, B.J., P.J. Bowman, A.J. Chamberlain, and M.E. Goddard 2009. Invited review: Genomic selection in dairy cattle: Progress and challenges. J. Dairy Sci. 92:433-443. https://doi.org/10.3168/ jds.2008-1646.

Höglund, J. K., G. Sahana, R. F. Brøndum, B. Guldbrandtsen, B. Buitenhuis, and M. S. Lund. 2014. Fine mapping QTL for female fertility on BTA04 and BTA13 in dairy cattle using HD SNP and sequence data. BMC Genomics 15:790. https://doi.org/10.1186/ 1471-2164-15-790.

Kang, H.M., N.A. Zaitlen, and E. Eskin. 2010. EMINIM: An adaptive and memory-efficient algorithm for genotype imputation. J. Comput. Biol. 17:547-560. https://doi.org/10.1089/cmb.2009.0199.

Legarra, A., C. Robert-Granié, P. Croiseau, F. Guillaume, and S. Fritz. 2011. Improved Lasso for genomic selection. Genet Res (Camb) 93:77-87. https://doi.org/10.1017/S0016672310000534.

Liu, Z., F. R. Seefried, F. Reinhardt, S. Rensing, G. Thaller, and R. Reents. 2011. Impacts of both reference population size and inclusion of a residual polygenic effect on the accuracy of genomic prediction. Genet. Sel. Evol. 43:19. https://doi.org/10.1186/1297 -9686-43-19.

Lund, M. S., A. P. W. De Roos, A. G. De Vries, T. Druet, V. Ducrocq, S. Fritz, F. Guillaume, B. Guldbrandtsen, Z. Liu, R. Reents, C. Schrooten, F. Seefried, and G. Su. 2011. A common reference population from four European Holstein populations increases reliability of genomic predictions. Genet. Sel. Evol. 43:43. https://doi .org/10.1186/1297-9686-43-43.

Ma, P., M. S. Lund, U. S. Nielsen, G. P. Aamand, and G. Su. 2015. Single-step genomic predictions improved prediction reliability and eliminated bias of the prediction trend in Danish Jersey. J. Dairy Sci. 98:9026-9034. https://doi.org/10.3168/jds.2015-9703.

Madsen, P., G. Su, R. Labouriau, and O. F. Christensen. 2010. DMUA package for analyzing multivariate mixed models. Page 732 in Proc. 9th World Congr. Genet. Appl. Livest. Prod., Leipzig, Germany. Gesellschaft für Tierzuchtwissenschaft e.V., Bonn, Gemany.

Meuwissen, T. H. E., B. J. Hayes, and M. E. Goddard. 2001. Prediction of total genetic value using genome-wide dense marker maps. Genetics 157:1819-1829.

Neves, H. H. R., R. Carvalheiro, A. M. P. O'Brien, Y. T. Utsunomiya, A. S. do Carmo, F. S. Schenkel, J. Sölkner, J. C. McEwan, C. P. Van Tassell, J. B. Cole, M. V. G. B. da Silva, S. A. Queiroz, T. S. Sonstegard, and J. F. Garcia. 2014. Accuracy of genomic predictions in Bos indicus (Nellore) cattle. Genet. Sel. Evol. 46:17. https: //doi.org/10.1186/1297-9686-46-17.

Ni, G.. D. Cavero, A. Fangmann, M. Erbe, and H. Simianer. 2017. Whole-genome sequence-based genomic prediction in laying chickens with different genomic relationship matrices to account for genetic architecture. Genet. Sel. Evol. 49:8. https://doi.org/10.1186/ s12711-016-0277-y.

Schaeffer, L. R. 2006. Strategy for applying genome-wide selection in dairy cattle. J. Anim. Breed. Genet. 123:218-223. https://doi.org/ 10.1111/j.1439-0388.2006.00595.x.

Steiger, J. H. 1980. Tests for comparing elements of a correlation matrix. Psychol. Bull. 87:245-251.

Su, G., R. F. Brøndum, P. Ma, B. Guldbrandtsen, G. P. Aamand, and M. S. Lund. 2012. Comparison of genomic predictions using medium-density $(\sim 54,000)$ and high-density $(\sim 777,000)$ single nucleotide polymorphism marker panels in Nordic Holstein and Red Dairy Cattle populations. J. Dairy Sci. 95:4657-4665. https:/ /doi.org/10.3168/jds.2012-5379.

$\mathrm{Su}$, G., O. F. Christensen, L. Janss, and M. S. Lund. 2014a. Comparison of genomic predictions using genomic relationship matrices built with different weighting factors to account for locus-specific 
variances. J. Dairy Sci. 97:6547-6559. https://doi.org/10.3168/jds .2014-8210.

Su, G., B. Guldbrandtsen, G. P. Aamand, I. Strandén, and M. S. Lund. 2014b. Genomic relationships based on X chromosome markers and accuracy of genomic predictions with and without $\mathrm{X}$ chromosome markers. Genet. Sel. Evol. 46:47. https://doi.org/10 .1186/1297-9686-46-47.

van Binsbergen, R., M. P. L. Calus, M. C. A. M. Bink, F. A. van Eeuwijk, C. Schrooten, and R. F. Veerkamp. 2015. Genomic prediction using imputed whole-genome sequence data in Holstein Friesian cattle. Genet. Sel. Evol. 47:71. https://doi.org/10.1186/ s12711-015-0149-x.

van den Berg, I., D. Boichard, and M. S. Lund. 2016. Sequence variants selected from a multi-breed GWAS can improve the reliability of genomic predictions in dairy cattle. Genet. Sel. Evol. 48:83. https://doi.org/10.1186/s12711-016-0259-0.

VanRaden, P. M. 2008. Efficient methods to compute genomic predictions. J. Dairy Sci. 91:4414-4423. https://doi.org/10.3168/jds .2007-0980.

VanRaden, P. M., C. P. Van Tassell, G. R. Wiggans, T. S. Sonstegard, R. D. Schnabel, J. F. Taylor, and F. S. Schenkel. 2009. Invited review: Reliability of genomic predictions for North American Holstein bulls. J. Dairy Sci. 92:16-24. https://doi.org/10.3168/jds .2008-1514.

Wu, X., M. S. Lund, G. Sahana, B. Guldbrandtsen, D. Sun, Q. Zhang, and G. Su. 2015a. Association analysis for udder health based on SNP-panel and sequence data in Danish Holsteins. Genet. Sel. Evol. 47:50. https://doi.org/10.1186/s12711-015-0129-1.

Wu, X., M. S. Lund, D. Sun, Q. Zhang, and G. Su. 2015b. Impact of relationships between test and training animals and among training animals on reliability of genomic prediction. J. Anim. Breed. Genet. 132:366-375. https://doi.org/10.1111/jbg.12165.

Yang, J., B. Benyamin, B. P. McEvoy, S. Gordon, A. K. Henders, D. R. Nyholt, P. A. Madden, A. C. Heath, N. G. Martin, G. W. Montgomery, M. E. Goddard, and P. M. Visscher. 2010. Common SNPs explain a large proportion of the heritability for human height. Nat. Genet. 42:565-569. https://doi.org/10.1038/ng.608.

Zhou, L., M. S. Lund, Y. Wang, and G. Su. 2014. Genomic predictions across Nordic Holstein and Nordic Red using the genomic best linear unbiased prediction model with different genomic relationship matrices. J. Anim. Breed. Genet. 131:249-257. https://doi.org/10 $.1111 /$ jbg. 12089 . 